\title{
CD133 mediates the TGF- $\beta 1$-induced activation of the PI3K/ERK/P70S6K signaling pathway in gastric cancer cells
}

\author{
YOULONG ZHU ${ }^{1 *}$, FEIFEI KONG ${ }^{2 *}$, CAIHUA ZHANG ${ }^{1}$, CHENG MA $^{1}$, \\ HONG XIA $^{1}$, BIN QUAN ${ }^{1}$ and HUAIXIN CUI ${ }^{1}$ \\ ${ }^{1}$ Department of Second Gastrointestinal Surgery, Xuzhou Central Hospital, School of Medicine, Southeast University; \\ ${ }^{2}$ Department of Oncology, The Affiliated Hospital of Xuzhou Medical University, Xuzhou, Jiangsu 221000, P.R. China
}

Received March 5, 2017; Accepted October 3, 2017

DOI: $10.3892 / \mathrm{ol} .2017 .7163$

\begin{abstract}
Cluster of differentiation (CD)133 has been reported to be involved in the activation of the extracellular signal-regulated kinase (ERK) signaling pathway in different types of cancer cells. CD133 has been reported to be involved in the activation of the ERK signaling pathway in various cancer cells. Transforming growth factor (TGF)- $\beta 1$ has also been reported to mediate the activation of the ERK signaling pathway. In addition, TGF- $\beta 1$ has been previously shown to mediate the activation of the ERK signaling pathway. Hence, the present study investigated the function of CD133 in the TGF- $\beta 1$-induced activation of the ERK/P70S6K signaling pathway in human gastric cancer (GC) cells. To this end, GC cell lines SGC7901 and MKN45 were treated with TGF- $\beta 1$. The expression of CD133, phospho-ERK (p-ERK) and phospho-P70S6 kinase (p-P70S6K) was upregulated in the cells treated with TGF- $\beta 1$, while the expression of ERK and P70S6K was not altered. To investigate whether CD133 is involved in the TGF- $\beta 1$-induced activation of the ERK/P70S6K signaling pathway in GC cells, immunomagnetic cell sorting was employed to isolate $\mathrm{CD} 133^{+} \mathrm{GC}$ cells, and a CD133-expression construct or CD133-targeting small interfering ribonucleic acids were transfected into cells to modulate the expression of CD133. Subsequently, the expression of CD133, ERK, p-ERK, P70S6K, and p-P70S6K was analyzed by western blotting. The $\mathrm{CD} 133^{+}$cells displayed a high expression of p-ERK and p-P70S6K. Furthermore, SGC7901 GC cells were treated with U0126, an inhibitor of the
\end{abstract}

Correspondence to: Mr. Bin Quan or Professor Huaixin Cui, Department of Second Gastrointestinal Surgery, Xuzhou Central Hospital, School of Medicine, Southeast University, Xuzhou, Jiangsu 221000, P.R. China

E-mail: zhuyoulong1987@126.com

E-mail:konfeifei@163.com

*Contributed equally

Key words: cluster of differentiation 133, gastric cancer, extracellular signal-regulated kinase/P70S6K
ERK signaling pathway, to assess whether CD133 is upstream of ERK/P70S6K. The results showed that the expression of p-ERK and p-P70S6K was downregulated in the cells treated with U0126, while the expression of CD133 remained unaltered. The above preliminary results showed that CD133 likely mediates the TGF- $\beta 1$-induced activation of the ERK/P70S6K signaling pathway in human GC cells. To further understand the mechanism of regulation of the ERK/P70S6K signaling pathway by CD133, the expression of CD133 was modulated by transfecting cells with CD133-expression constructs or CD133-targeting small interfering ribonucleic acids. Results indicated that overexpression and silencing of CD133 directly increased and decreased the expression of p-ERK and p-P70S6K, respectively. Therefore, we hypothesized that CD133 mediates the TGF- $\beta 1$-induced activation of the PI3K/ERK/P70S6K signaling pathway in human GC cells.

\section{Introduction}

Stomach adenocarcinoma or gastric cancer (GC) is the fourth most common cancer and the second highest cause of cancer-related mortality worldwide (1). Despite improvements in surgical and adjuvant treatment approaches, the prognosis of patients with GC continues to be dismal, with a 5-year overall survival lower than $25 \%$ (2). The success of personalized therapy depends on the identification and inhibition of the oncogene(s) on which the tumor depends. Thus, it is of great clinical significance to identify genes that determine the severity of GC and assess their predictive value in the prognosis of GC $(3,4)$. Previous studies have shown that most GC patients die due to metastasis and treatment failure. Therefore, there is an urgent need to improve the understanding of the mechanisms that lead to GC so that new treatment strategies may be developed to target the associated pathways. Accumulating reports have shown that the extracellular signal-regulated kinase (ERK) signaling pathway is associated with increased motility, invasion and metastasis of cancer cells. Moreover, the ERK signaling pathway is frequently found to be amplified in GC (5-8). Since ERK signaling is also controlled by spatiotemporal regulatory mechanisms $(9,10)$, it is of great interest to determine if there is a key gene that contributes to the deregulation of ERK in GC. Hence, elucidating the mechanism of activation of the ERK signaling pathway will further provide 
a novel approach to treat GC and thereby improve the survival of GC patients.

Interestingly, several studies have reported that cluster of differentiation (CD)133 contributes to the activation of the ERK signaling pathway (11). The transmembrane protein CD133 is of particular interest and a controversial subject. However, the physiological function of CD133 remains unclear. CD133 is the most commonly expressed cancer stem cell (CSC) marker in various cancer types such as colon, lung, brain, pancreas, and GC (12-15). It has been shown that $\mathrm{CD} 133^{+}$cells exhibit a higher degree of activation of the ERK signaling pathway than CD133- cells (8). However, the biological function of CD133 in the activation of the ERK signaling pathway in GC cells is still unknown.

CD133 has been reported to be a useful marker for predicting recurrence and chemotherapy efficacy in not only breast cancer but also GC $(16,17)$. Accumulating evidence strongly suggests the functional association of $\mathrm{CD} 133^{+} \mathrm{CSC}$ with the ERK signaling pathway. $\mathrm{CD}_{133^{+}}$tumor cells derived from hepatoma (18), colon cancer (19), melanoma (20), malignant peripheral nerve sheath tumor (21) and neuroblastoma (22) samples consistently displayed increased phospho-ERK (p-ERK) levels compared with matched CD133- tumor cells. In addition, the overexpression of CD133 has been shown to promote the phosphorylation of Erk in U87MG human glioblastoma cells. These results strongly imply that CD133 facilitates the activation of the ERK signaling pathway in many tumor cells. However, the role of CD133 in GC cells has not yet been studied.

The transforming growth factor (TGF)- $\beta$ family plays a pivotal role in regulating a variety of cellular processes such as differentiation, proliferation and apoptosis. TGF- $\beta 1$ has also been shown to mediate the activation of a certain downstream targets of the PI3K signaling pathway, such as Jnk and Erk (23-26). Moreover, the TGF- $\beta 1$ signaling pathway has been shown to regulate the function of $\mathrm{CD} 133^{+} \mathrm{CSC}$ in human brain tumors (27). Vangipuram demonstrated that TGF- $\beta 1$ stimulation can enhance CD133 expression in a time- and dose-dependent manner in Huh7 HCC cells (28).

In the present study, we investigated whether CD133 can mediate the TGF- $\beta 1$-induced activation of the ERK/P70S6K signaling pathway in GC cells. This study may provide insights into the molecular mechanism(s) responsible for the activation of the TGF- 31 -mediated ERK/P70S6K signaling pathway and enable the development of effective anticancer therapies.

\section{Materials and methods}

Chemicals. TGF- $\beta 1$ was purchased from PeproTech, Inc. (Rocky Hill, NJ, USA), and U0126, a small molecular inhibitor of the ERK pathway, was purchased from Sigma-Aldrich, Inc. (St. Louis, MO, USA).

Cell lines and cultures. The human GC cell lines MKN45 and SGC7901 were provided by the Shanghai Institute of Cell Biology, CAS (Shanghai, China). The cells were cultured in Roswell Park Memorial Institute (RPMI) 1640 culture medium (HyClone, Logan, UT, USA) supplemented with $100 \mathrm{~g} / \mathrm{ml}$ streptomycin, $100 \mathrm{U} / \mathrm{ml}$ penicillin, and $10 \%$ fetal bovine serum (HyClone) at $37^{\circ} \mathrm{C}$ in a humidified environment containing $5 \%$ carbon dioxide.
Immunomagnetic cell sorting. The cells were subcultured every 2 to 3 days. The third to fifth subcultures were harvested, and the CD133 immunomagnetic cell sorting kit (Miltenyi Biotec, Bergisch Gladbach, Germany) was used to isolate the $\mathrm{CD} 133^{+} \mathrm{GC}$ cells. The $\mathrm{CD} 133^{+}$cells were maintained in serum-free RPMI 1640 medium at $37^{\circ} \mathrm{C}$ in a humidified environment containing $5 \%$ carbon dioxide $(29,30)$.

Transient transfection of CD133 small interfering RNA (siRNA). CD133-specific siRNA oligonucleotides were purchased based on the CD133 gene sequence (Shanghai GenePharma Co., Ltd., Shanghai, China). The sequences of the double-stranded siRNA oligonucleotides were 5'-GUC CUUCCUAUAGAACAAUTT-3' (sense) and 5'-AUUGUU CUAUAGGAAGGACTT-3' (antisense). The negative control siRNA sequences were 5'-UUCUCCGAACGUGUCACG UTT-3' (sense) and 5'-ACGUGACACGUUCGGAGAATT-3' (antisense). Human GC SGC7901 cells were transfected with siRNA (100 nM) using Lipofectamine 2000 (Invitrogen, Carlsbad, CA, USA) according to the manufacturer's protocol.

Stable transfection of CD133. A plasmid extraction kit (Qiagen, Düsseldorf, Germany) was used to extract the CD133 complementary deoxyribonucleic acid (cDNA)-encoding plasmid according to the manufacturer's protocol. The human GC SGC7901 cells, which have been confirmed to have low CD133 expression, were stably transfected using Lipofectamine ${ }^{\circledR}$ LTX Reagent (Invitrogen, Tokyo, Japan) in accordance with the manufacturer's instructions.

Western blotting and antibodies. Quantified protein lysates were resolved via sodium dodecyl sulfate-polyacrylamide gel electrophoresis, transferred onto polyvinylidene difluoride membranes (Millipore, Billerica, MA, USA), and incubated with primary antibodies (CD133/1 mouse mAb 1:100, Miltenyi Biotec; and phospho-P70S6 kinase (p-P70S6K), P70S6K, p-ERK and ERK, rabbit $m A b$ 1:1,000, Cell Signaling Technology, Inc., Boston, MA, USA), followed by incubation with the appropriate HRP-conjugated secondary antibodies (1:2,000; Jackson, Mukilteo, WA, USA) at room temperature. Immunoreactive proteins were detected using an enhanced chemiluminescence detection kit (Amersham Biosciences, Inc., Piscataway, NJ, USA).

Statistical analyses. Student's t-test and ANOVA were used to compare the results, when appropriate. All statistical analyses were performed using the software SPSS 13.0 (SPSS, Inc., Chicago, IL, USA). Values of $\mathrm{P}<0.05$ were considered statistically significant.

\section{Results}

TGF- $\beta 1$ upregulates the level of CD133 and activates the ERK/P70S6K signaling pathway. Recent studies have shown that CD133 expression is regulated by microenvironmental changes within the CSC niche $(31,32)$. We hypothesized that CD133 expression is regulated by known growth factors, such as TGF- $\beta 1$, which are highly expressed in GC. To confirm our hypothesis, SGC7901 and MKN45 cells were treated with $5 \mathrm{ng} / \mathrm{ml}$ TGF- $\beta 1$ and analyzed via immunoblotting. Fig. 1A-D 
A

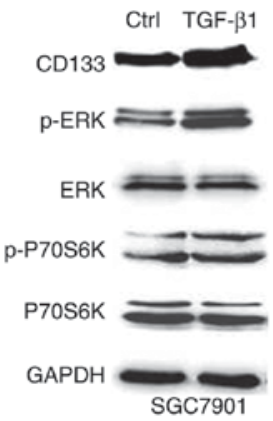

B

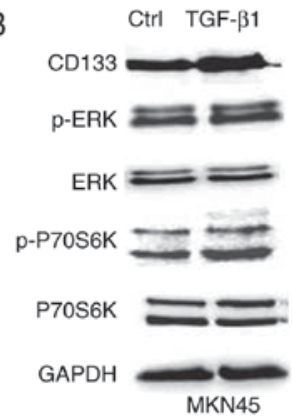

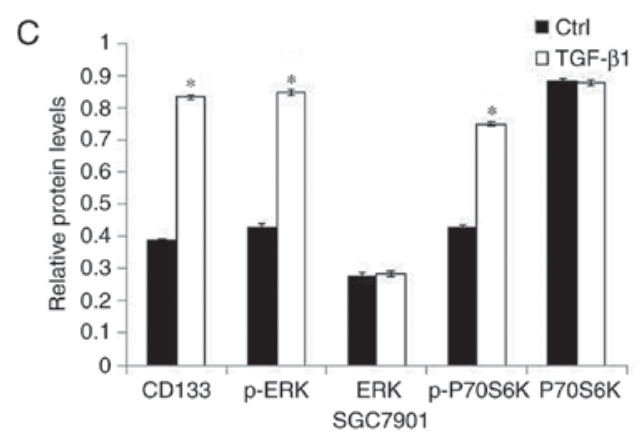

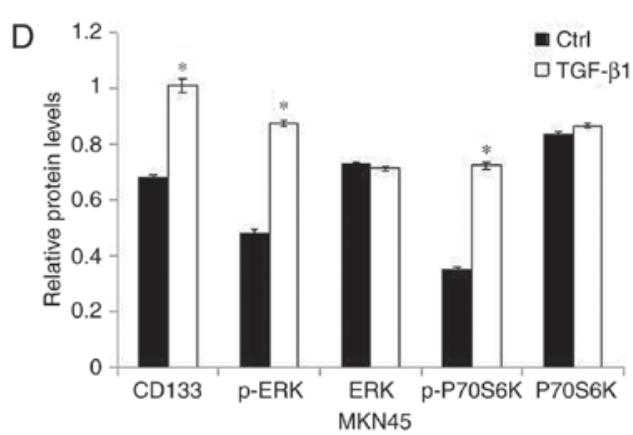

Figure 1. TGF- $\beta 1$ upregulates the level of CD133 and activates the ERK/P70S6K signaling pathway. (A and B) Expression of CD133, p-ERK, ERK, p-P70S6K and P70S6K was determined in SGC7901 and MKN45 cells by western blotting. Glyceraldehyde 3-phosphate dehydrogenase protein was used as an internal control. (C and D) Quantification of the target protein bands relative to GAPDH levels is shown in the panels, "P<0.05 vs. Ctrl. TGF, transforming growth factor; $\mathrm{CD}$, cluster of differentiation; ERK, extracellular signal-regulated kinase; p-ERK, phospho-ERK; p-P70S6K, phospho-P70S6 kinase.

A
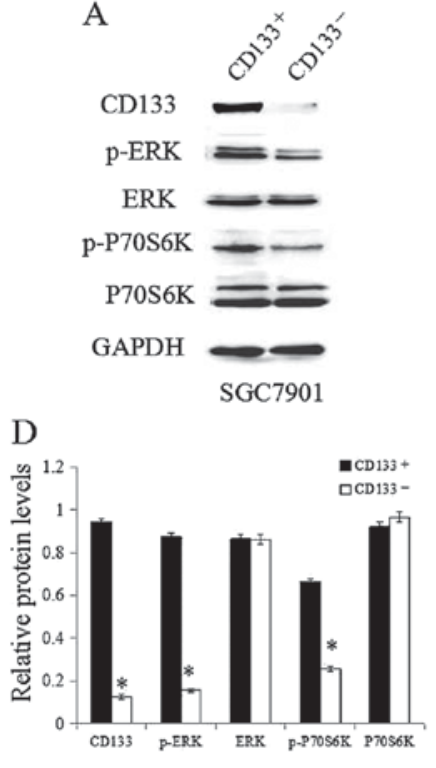

SGC7901
B

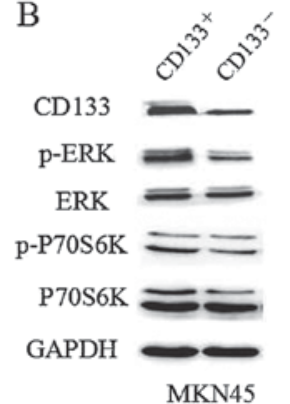

$\mathrm{C}$

CD133

p-ERK - -

$\mathrm{ERK}=$

$\mathrm{p}-\mathrm{P} 70 \mathrm{~S} 6 \mathrm{~K}=$

P70S6K $=$

GAPDH

SGC7901

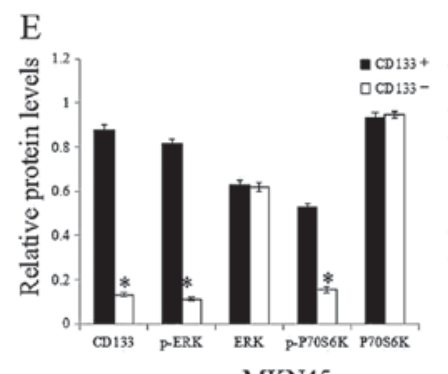

MKN45

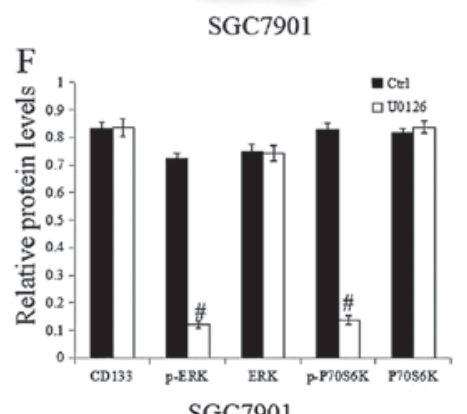

Figure 2. CD133 ${ }^{+} \mathrm{GC}$ cells display a greater extent of activation of ERK/P70S6K signaling. CD133 ${ }^{+}$and CD133- GC cells were isolated. (A and B) Western blotting was used to confirm the expression levels of CD133, p-ERK, ERK, p-P70S6K and P70S6K. (C) The effect of U0126 treatment on the expression of CD133, p-ERK, ERK, p-P70S6K and P70S6K. (D and E) Quantification of the target protein bands relative to GAPDH levels is shown in the panels. (F) Quantification of the target protein bands relative to GAPDH levels is shown in the panels " $\mathrm{P}<0.05$ vs. CD133 ${ }^{+}$; ${ }^{*} \mathrm{P}<0.05$ vs. Ctrl. CD, cluster of differentiation; GC, gastric cancer; ERK, extracellular signal-regulated kinase; p-ERK, phospho-ERK; p-P70S6K, phospho-P70S6 kinase.

shows that the expression of CD133 protein was enhanced by TGF- $\beta 1$ treatment. In addition, the expression level of $\mathrm{p}-\mathrm{ERK}$ and p-P70S6K was induced in GC cells treated with TGF- $\beta 1$, while the expression of ERK and P70S6K was not affected.

$C D 133^{+}$GC cells display a higher degree of activation of ERK/P70S6K signaling. To test whether TGF- $\beta 1$ activates the
ERK/P70S6K pathway by regulating CD133 expression, we examined ERK, p-ERK, P70S6K and p-P70S6K expression levels in $\mathrm{CD}_{133^{+}}$and CD133- GC cells. As shown in Fig. 2A, B, $\mathrm{D}$ and $\mathrm{E}$, there was no significant difference in the expression of total ERK and P70S6K between CD133+ and CD133- cells, but the expression of p-ERK and p-P70S6K was significantly higher in the $\mathrm{CD} 133^{+}$cells compared to that in the CD133- cells. 

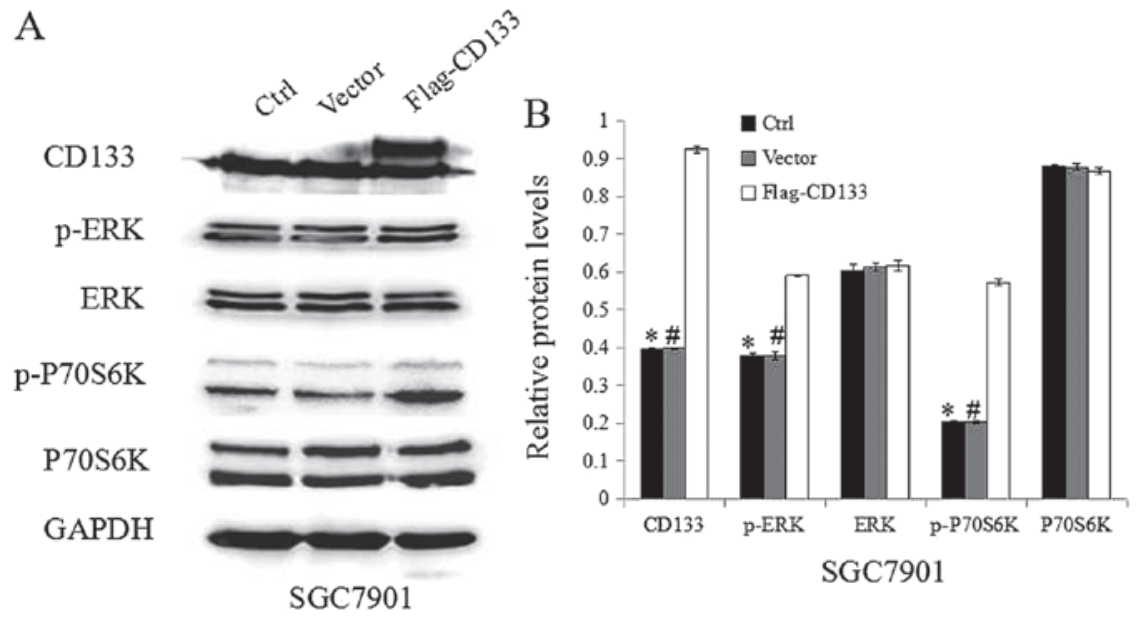

Figure 3. CD133 activation enhances ERK/P70S6K activity. Cells were transfected with either empty pcDNA vector or CD133-expression vector. (A) Western blotting was performed to investigate the expression of CD133, p-ERK, ERK, p-P70S6K and P70S6K. (B) Quantification of the target protein bands relative to GAPDH levels is shown in the panels, ${ }^{*} \mathrm{P}<0.05$ vs. Ctrl; ${ }^{\#} \mathrm{P}<0.05$ vs. vector. $\mathrm{CD}$, cluster of differentiation; ERK, extracellular signal-regulated kinase; $\mathrm{p}$-ERK, phospho-ERK; p-P70S6K, phospho-P70S6 kinase.

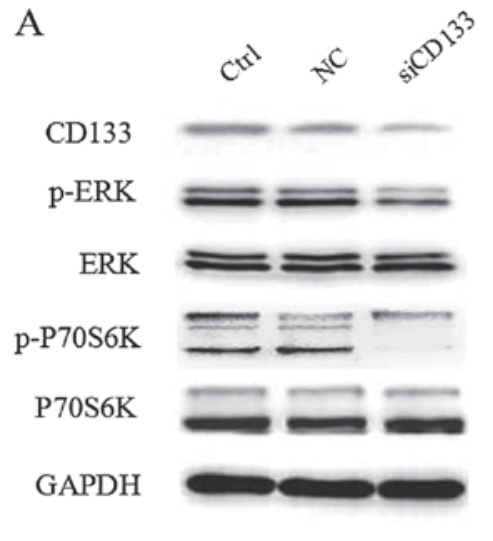

SGC7901

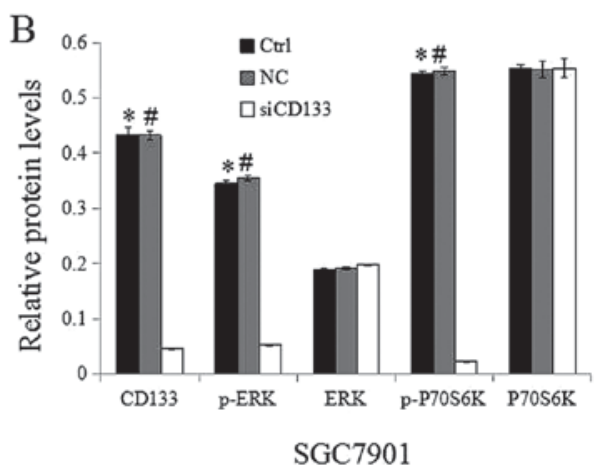

Figure 4. Inhibition of CD133 in SGC7901 GC cells inhibits the activation of ERK/P70S6K signaling. (A) Protein expression was analyzed via western blotting using antibodies against CD133, p-ERK, ERK, p70S6K, p-p70S6K, and glyceraldehyde 3-phosphate dehydrogenase. (B) Quantification of the target protein bands relative to GAPDH levels is shown in the panels, ${ }^{\mathrm{P}}<0.05$ vs. Ctrl; " $\mathrm{P}<0.05$ vs. NC. CD, cluster of differentiation; GC, gastric cancer; ERK, extracellular signal-regulated kinase; p-ERK, phospho-ERK; p-P70S6K, phospho-P70S6 kinase.

To clarify whether CD133 is upstream of the ERK/P70S6K signaling pathway, SGC7901 GC cells were treated with the specific inhibitor of the ERK pathway U0126. Western blotting showed that U0126 treatment clearly decreased p-ERK and p-P70S6K expression, while the expression of CD133 was unchanged (Fig. 2C and F). Taken together, our results indicate that CD133 is likely upstream of the ERK/P70S6K signaling pathway. Given that TGF- $\beta 1$ both activates the ERK/P70S6K pathway and upregulates CD133 expression, CD133 might be a mediator of the TGF- $\beta 1$-induced activation of the ERK/P70S6K signaling pathway.

CD133 activation enhances ERK/P70S6K activity. To confirm the role of CD133 in the TGF- $\beta 1$-induced activation of ERK/P70S6K signaling, the expression of CD133 was increased by transfecting the CD133-expression construct into SGC7901 cancer cells. Western blotting demonstrated that CD133 expression was clearly upregulated in the CD133-overexpressing cells compared with that in the vector-transfected cells (Fig. 3A and B). Meanwhile, the expression of p-ERK and p-P70S6K was upregulated in the CD133-overexpressing cells compared to that in the empty vector-transfected cells, while the expression of ERK and P70S6K was not affected.

Inhibition of CD133 in SGC7901 GC cells inhibits the activation of ERK/P70S6K signaling. To further confirm the significance of CD133, siRNAs targeting CD133 were used. As indicated in Fig. 4A and B, the expression of CD133 in GC cells was successfully inhibited by siRNAs targeting CD133. Western blotting showed that the downregulation of CD133 contributed to a reduction in the level of p-ERK and p-P70S6K in cells transfected with siRNAs targeting CD133 compared to that in cells transfected with control siRNAs. In contrast, the expression of ERK and P70S6K was not significantly altered after CD133 inhibition. Taken together, CD133 likely plays an important role in the TGF- $\beta 1$-induced activation of the ERK/P70S6K signaling pathway. 


\section{Discussion}

CD133 is a transmembrane protein that is well adapted to participate in ERK signaling regulated by TGF- $\beta 1$.

Better understanding of the relevance and function of CSCs may provide novel insights into the underlying mechanislms and possible targets for GC therapies. Increasing evidence has demonstrated that in addition to being a biomarker in tumors, CD133 regulates the growth and development of tumor cells. Recently, CD133 has been reported to be involved in the activation of the ERK signaling pathway in various cancer cells (18-22). Moreover, increasing evidence has indicated that the enhanced motility, invasion and metastasis of cancer cells are associated with the ERK signaling pathway. Although the activation of the ERK signaling pathway has been reported to be associated with GC, we are the first to report that CD133 involved in the activation of the ERK signaling pathway induced by TGF- $\beta 1$ in GC. Our current study demonstrates the correlation between CD133 and the TGF- $\beta 1$-mediated activation of the ERK/P70S6K signaling pathway in GC cells.

In the current study, CD133 protein expression was induced by TGF- $\beta 1$ treatment. In addition, the expression level of p-ERK and p-P70S6K was upregulated in GC cells treated with TGF- $\beta 1$, while the expression of ERK and P70S6K was not changed. The above results showed that TGF- $\beta 1$ might activate CD133 as well as the ERK/P70S6K signaling pathway. However, the correlation among TGF- $\beta 1$, CD133 and ERK/P70S6K signaling pathway remained unclear.

To test whether TGF- $\beta 1$ activates the ERK/P70S6K pathway by regulating CD133 expression, we examined the expression of ERK, p-ERK, P70S6K and p-P70S6K in CD133+ and CD133- GC cells. As demonstrated, there was no obvious difference in total ERK and P70S6K levels between CD133 ${ }^{+}$ and CD133- cells, but p-ERK and p-P70S6K levels were significantly higher in the $\mathrm{CD} 133^{+}$cells compared to those in the CD133- cells. To clarify whether CD133 is upstream of the ERK/P70S6K signaling pathway, SGC7901 GC cells were treated with the specific inhibitor of the ERK signaling pathway U0126. Western blotting showed that U0126 treatment clearly decreased the expression of p-ERK and p-P70S6K, while the expression of CD133 was unchanged. Taken together, our results indicate that CD133 is likely upstream of the ERK/P70S6K signaling pathway. Given that TGF- $\beta 1$ activates the ERK/P70S6K pathway and upregulates CD133 expression, CD133 is the mediator of the TGF- $\beta 1$-induced activation of the ERK/P70S6K signaling pathway.

To confirm the role of CD133 in the TGF- $\beta 1$-induced activation of ERK/P70S6K signaling, gene modulation was used. In our current study, the function of CD133 on the activation of the ERK/P70S6K signaling pathway was confirmed by upregulating and downregulating CD133 in SGC7901 cells. It was found that silencing CD133 in cells via CD133-siRNAs resulted in a reduction in the level of p-ERK and p-P70S6K compared to that in control siRNA-transfected cells. In addition, the activation of CD133 increased the expression of p-ERK and p-P70S6K in cells transfected with siRNAs targeting CD133 compared to that in cells transfected with control siRNA, while the expression of ERK and P70S6K was not affected. Taken together, these observations clearly suggest that CD133 plays an important role in the TGF- $\beta 1$-induced activation of the ERK/P70S6K signaling pathway.

In conclusion, the results of the present study suggest that concurrent blocking of CD133 and the ERK/P70S6K pathway might be an effective approach for improving the prognosis of GC patients. In addition, our results provide important avenues for future research in GC. However, studies investigating the association between CD133 and the TGF- $\beta 1$-induced activation of the ERK/P70S6K signaling pathway in GC cells using human GC specimens and animal models are necessary to validate the usefulness of this approach. What's more, a limitation of the present study is that we did not assess whether TGF-beta1-induced CD133 and subsequently activation of PI3K enhances cell growth. In the future, we will conduct a functional study aimed to demonstrate the correlation between the PI3K pathway and cell growth. Furthermore, we did not prove whether the activation of the PI3K pathway by CD133 is mediated by the phosphorylation of the regulatory subunits p85 and/or p110. Obviously, the absence of data on PI3K/PTEN and PI3K/AKT signaling was also a limitation of this study. And our future study will solve the above problems.

\section{Acknowledgements}

The present study was supported by funds from the Hospital Foundation of Xuzhou Central Hospital, which is affiliated with the Medical College of Southeast University (grant no. XZS201673).

\section{References}

1. Coupland VH, Lagergren J, Lüchtenborg M, Jack RH, Allum W, Holmberg L, Hanna GB, Pearce N and Møller H: Hospital volume, proportion resected and mortality from oesophageal and gastric cancer: A population-based study in England, 2004-2008. Gut 62: 961-966, 2013.

2. Camargo MC, Kim WH, Chiaravalli AM, Kim KM, Corvalan AH, Matsuo K, Yu J, Sung JJ, Herrera-Goepfert R, MenesesGonzalez F, et al: Improved survival of gastric cancer with tumour Epstein-Barr virus positivity: An international pooled analysis. Gut 63: 236-243, 2014.

3. Shibata W, Ariyama H, Westphalen CB, Worthley DL, Muthupalani S, Asfaha S, Dubeykovskaya Z, Quante M, Fox JG and Wang TC: Stromal cell-derived factor-1 overexpression induces gastric dysplasia through expansion of stromal myofibroblasts and epithelial progenitors. Gut 62: 192-200, 2013.

4. Wang S, Wu X, Zhang J, Chen Y, Xu J, Xia X, He S, Qiang F, $\mathrm{Li} \mathrm{A}$, Shu Y, et al: CHIP functions as a novel suppressor of tumour angiogenesis with prognostic significance in human gastric cancer. Gut 62: 496-508, 2013.

5. Paterson AL, Shannon NB, Lao-Sirieix P, Ong CA, Peters CJ, O'Donovan M and Fitzgerald RC: A systematic approach to therapeutic target selection in oesophago-gastric cancer. Gut 62: 1415-1424, 2013.

6. Jiang M, Qiu Z, Zhang S, Fan X, Cai X, Xu B, Li X, Zhou J, Zhang X, Chu Y, et al: Elevated O-GlcNAcylation promotes gastric cancer cells proliferation by modulating cell cycle related proteins and ERK 1/2 signaling. Oncotarget 7: 61390-61402, 2016.

7. Zhou Q, Wang X, Yu Z, Wu X, Chen X, Li J, Zhu Z, Liu B and Su L: Transducin $(\beta)$-like $1 \mathrm{X}$-linked receptor 1 promotes gastric cancer progression via the ERK1/2 pathway. Oncogene 36: 1873-1886, 2017.

8. Zhang X, Hua R, Wang X, Huang M, Gan L, Wu Z, Zhang J, Wang H, Cheng Y, Li J and Guo W: Identification of stem-like cells and clinical significance of candidate stem cell markers in gastric cancer. Oncotarget 7: 9815-9831, 2016.

9. Kholodenko BN, Hancock JF and Kolch W: Signalling ballet in space and time. Nat Rev Mol Cell Biol 11: 414-426, 2010. 
10. Kong X, Qian J, Chen LS, Wang YC, Wang JL, Chen H, Weng YR, Zhao SL, Hong J, Chen YX, et al: Synbindin in extracellular signal-regulated protein kinase spatial regulation and gastric cancer aggressiveness. J Natl Cancer Inst 105: 1738-1749, 2013.

11. Dong L, Qi N, Ge RM, Cao CL, Lan F and Shen L: Overexpression of CD133 promotes the phosphorylation of Erk in U87MG human glioblastoma cells. Neurosci Lett 484: 210-214, 2010.

12. O'Brien CA, Pollett A, Gallinger S and Dick JE: A human colon cancer cell capable of initiating tumour growth in immunodeficient mice. Nature 445: 106-110, 2007.

13. Eramo A, Lotti F, Sette G, Pilozzi E, Biffoni M, Di Virgilio A Conticello C, Ruco L, Peschle C and De Maria R: Identification and expansion of the tumorigenic lung cancer stem cell population. Cell Death Differ 15: 504-514, 2008.

14. Singh SK, Clarke ID, Terasaki M, Bonn VE, Hawkins C, Squire J and Dirks PB: Identification of a cancer stem cell in human brain tumors. Cancer Res 63: 5821-5828, 2003.

15. Hermann PC, Huber SL, Herrler T, Aicher A, Ellwart JW, Guba M, Bruns CJ and Heeschen C: Distinct populations of cancer stem cells determine tumor growth and metastatic activity in human pancreatic cancer. Cell Stem Cell 1: 313-323, 2007.

16. Aomatsu N, Yashiro M, Kashiwagi S, Takashima T, Ishikawa T Ohsawa M, Wakasa K and Hirakawa K: CD133 is a useful surrogate marker for predicting chemosensitivity to neoadjuvant chemotherapy in breast cancer. PLoS One 7: e 45865, 2012.

17. Zhu Y, Yu J, Wang S, Lu R, Wu J and Jiang B: Overexpression of CD133 enhances chemoresistance to 5-fluorouracil by activating the PI3K/Akt/p70S6K pathway in gastric cancer cells. Oncol Rep 32: 2437-2444, 2014.

18. Ding W, Mouzaki M, You H, Laird JC, Mato J, Lu SC and Rountree CB: $\mathrm{CD} 133^{+}$liver cancer stem cells from methionine adenosyl transferase 1 A-deficient mice demonstrate resistance to transforming growth factor (TGF)-beta-induced apoptosis. Hepatology 49: 1277-1286, 2009

19. Wang YK, Zhu YL, Qiu FM, Zhang T, Chen ZG, Zheng S and Huang J: Activation of Akt and MAPK pathways enhances the tumorigenicity of $\mathrm{CD} 133^{+}$primary colon cancer cells Carcinogenesis 31: 1376-1380, 2010.

20. El-Khattouti A, Selimovic D, Haïkel Y, Megahed M, Gomez CR and Hassan M: Identification and analysis of CD133(+) melanoma stem-like cells conferring resistance to taxol: An insight into the mechanisms of their resistance and response. Cancer Lett 343: 123-133, 2014

21. Borrego-Diaz E, Terai K, Lialyte K, Wise AL, Esfandyari T, Behbod F, Mautner VF, Spyra M, Taylor S, Parada LF, et al: Overactivation of Ras signaling pathway in CD133+ MPNST cells. J Neurooncol 108: 423-434, 2012.
22. Vangipuram SD, Wang ZJ and Lyman WD: Resistance of stem-like cells from neuroblastoma cell lines to commonly used chemotherapeutic agents. Pediatr Blood Cancer 54: 361-368, 2010.

23. Mucsi I, Skorecki KL and Goldberg HJ: Extracellular signal-regulated kinase and the small GTP-binding protein, Rac, contribute to the effects of transforming growth factor-betal on gene expression. J Biol Chem 271: 16567-16572, 1996.

24. Atfi A, Djelloul S, Chastre E, Davis R and Gespach C: Evidence for a role of Rho-like GTPases and stress-activated protein kinase/c-Jun N-terminal kinase (SAPK/JNK) in transforming growth factor beta-mediated signaling. J Biol Chem 272: 1429-1432, 1997.

25. Bakin AV, Tomlinson AK, Bhowmick NA, Moses HL and Arteaga CL: Phosphatidylinositol 3-kinase function is required for transforming growth factor beta-mediated epithelial to mesenchymal transition and cell migration. J Biol Chem 275: 36803-36810, 2000.

26. Bhowmick NA, Ghiassi M, Bakin A, Aakre M, Lundquist CA, Engel ME, Arteaga CL and Moses HL: Transforming growth factor-beta1 mediates epithelial to mesenchymal transdifferentiation through a RhoA-dependent mechanism. Mol Biol Cell 12: 27-36, 2001

27. Visvader JE and Lindeman GJ: Cancer stem cells in solid tumours: Accumulating evidence and unresolved questions. Nat Rev Cancer 8: 755-768, 2008.

28. You H, Ding W and Rountree CB: Epigenetic regulation of cancer stem cell marker CD133 by transforming growth factor-beta. Hepatology 51: 1635-1644, 2010.

29. Lu RQ, Wu JG, Zhou GC, Jiang HG, Yu JW and Jiang BJ: Sorting of CD133(+) subset cells in human gastric cancer and the identification of their tumor initiating cell-like properties. Zhonghua Wei Chang Wai Ke Za Zhi 15: 174-179, 2012 (In Chinese).

30. Takaishi S, Okumura T, Tu S, Wang SS, Shibata W, Vigneshwaran R, Gordon SA, Shimada Y and Wang TC: Identification of gastric cancer stem cells using the cell surface marker CD44. Stem Cells 27: 1006-1020, 2009.

31. Platet N, Liu SY, Atifi ME, Oliver L, Vallette FM, Berger F and Wion D: Influence of oxygen tension on CD133 phenotype in human glioma cell cultures. Cancer Lett 258: 286-290, 2007.

32. McCord AM, Jamal M, Shankavarum UT, Lang FF, Camphausen K and Tofilon PJ: Physiologic oxygen concentration enhances the stem-like properties of $\mathrm{CD} 133^{+}$human glioblastoma cells in vitro. Mol Cancer Res 7: 489-497, 2009. 